\title{
SOLUTION OF CONTACT PROBLEM FOR AN ARC CRACK USING HYPERSINGULAR INTEGRAL EQUATION
}

\author{
Y. Z. CHEN, X. Y. LIN and Z. X. WANG \\ Division of Engineering Mechanics \\ Jiangsu University, Zhenjiang \\ Jiangsu 212013, P. R. China \\ chens@ujs.edu.cn \\ N. M. A. NIK LONG \\ Mathematics Department, Faculty of Science \\ Institute for Mathematical Research \\ Universiti Putra Malaysia \\ 43400 Serdang, Selangor, Malaysia
}

Received 23 August 2007

Accepted 4 January 2008

\begin{abstract}
This paper investigates the contact problem for an arc crack, for example, under a remote compression. A hypersingular integral equation (HSIE) for curved cracks in plane elasticity is suggested. It is found that the direct usage of HSIE cannot solve the mentioned contact problem. For the contact problem, one must take necessary modifications for solving the HSIE. The main modified points are as follows. First, one should assume some portion along the crack under contact. The margin or the end of the contacted portion is determined by the vanishing normal contact stress at the margin point. In addition, it is found that a suggested quadrature rule in conjunction with the curve length method provides a very effective way to solve the HSIE. Finally, several numerical examples are given.
\end{abstract}

Keywords: Contact crack problem; hypersingular integration equation method; arc crack problem.

\section{Introduction}

The contact problems of cracks have not been solved very well in the past. This can be seen from an example. The solution of the stress intensity factors (SIFs) for an arc crack can be seen from Cotterell and Rice [1980] and Murakami [1987]. In the case of the remote traction $\sigma_{x y}^{\infty}=p$, the first mode SIF is negative at one crack tip from the mentioned solution. This means that the normal crack opening displacement (COD) must be overlapped near the crack tip. The overlapping of COD violates the physical situation of deformation. It is also clear that the mentioned solution cannot be used to the cases of $\sigma_{x}^{\infty}=-p$ and $\sigma_{y}^{\infty}=-p$. 
For a single line crack case, some contact crack problems were solved [Woo et al. (1988); Beghini and Bertini (1996)]. The contact problem for a single crack with a remote tension and a concentrated force was investigated [Chen (1999)]. Crack growth in brittle solids under compression was studied [Lauterbach and Gross (1998)]. Recently, the multiple crack problems under compression were studied [Basista and Gross (2000)]. Formulation of the problem was based on the assumption that the contact stresses acting on the crack faces are constant. Recently, the contact problem for the multiple cracks is solved [Chen (2004)]. Birinci and Cakiroglu [2003] studied a contact crack problem in an elastic layer. Those studies belong to the line crack case.

De Bremaecker et al. [2000a, 2000b] considered compressional fractures as a mixed complementary problem. Shear stresses along the crack face were investigated. Using singular integral equation method, the contact problem is viewed as a perturbation of a noncontacting problem [Helsing and Peters (2000)]. An arc crack problem in uniaxial loading was studied [Chao and Laws (1992); Chao and Rau (1995)].

It is found that the contact problem for an arc crack is more complicated, simply because the problem belongs to a problem of the undetermined boundary and the undetermined boundary traction. In a real contact problem, the normal component of COD should not be overlapped. However, in the usual singular integral equation of the type of dislocation versus the traction, the unknown function is the dislocation distribution. This is to say it is difficult to obtain the COD by using the mentioned singular integral equation.

The hypersingular integral equation (HSIE) for the curved crack is most suitable for the contact problems, because the unknown function in the equation is the COD. Comparatively speaking, the HSIE for the curved crack is novel, and it was proposed in a later time [Linkov and Mogilevskaya (1990, 1994); Chen (1993); Mogilevskaya (2000); Martin (2000); Linkov (2002)]. In addition, the involved hypersingular integral should be understood in the sense of finite part value [Hadamard (1923); Krenk (1975); Kaya and Erdogan (1987)]. In the integral equation, the COD is taken as unknown, and the boundary traction as right term.

In some cases, for example, the mentioned HSIE can be directly used to solve the problem for a slightly curved arc crack under the remote traction $\sigma_{x}^{\infty}=\sigma_{y}^{\infty}=p$. For a contact problem, one must take necessary modifications for solving the HSIE. The main modified points are as follows. First, one should assume some portion along the crack under contact. Alternatively speaking, the normal component of COD must vanish along those portions. The margin or the end of the contacted portion is determined by the vanishing normal contact stress at the margin point, which is shown by Eq. (20) later. In addition, it is found that a suggested quadrature rule in conjunction with the curve length method provides a very effective way to solve the HSIE [Mayrhofer and Fischer (1992); Chen (2003); Chen and Lin (2005)]. 
In this paper, for three remote loading cases, $\sigma_{x y}^{\infty}=p, \sigma_{x}^{\infty}=-p$, and $\sigma_{y}^{\infty}=-p$, the arc crack problem is solved with the computational results for SIFs.

\section{Hypersingular Integral Equation Method for the Contact Problem of an Arc Crack}

For convenience of reading this paper, a compact description for the derivation of the equation is carried out below, and the detail can be referred to [Chen (2003); Chen and Lin (2005)]. The complex variable function method plays an important role in plane elasticity [Muskhelishvili (1953)]. Fundamental of this method is introduced. In the method, the stresses $\left(\sigma_{x}, \sigma_{y}, \sigma_{x y}\right)$, the resultant forces $(X, Y)$, and the displacements $\left(u_{x}, u_{y}\right)$ are expressed in terms of complex potentials $\phi(z)$ and $\psi(z)$ such that

$$
\begin{gathered}
\sigma_{x}+\sigma_{y}=4 \operatorname{Re} \Phi(z), \\
\sigma_{y}-i \sigma_{x y}=2 \operatorname{Re} \Phi(z)+z \overline{\Phi^{\prime}(z)}+\overline{\Psi(z)} \\
f=-Y+i X=\phi(z)+z \overline{\phi^{\prime}(z)}+\overline{\psi(z)} \\
2 G\left(u_{x}+i u_{y}\right)=\kappa \phi(z)-z \overline{\phi^{\prime}(z)}-\overline{\psi(z)}
\end{gathered}
$$

where $G$ is the shear modulus of elasticity, $\kappa=(3-\nu) /(1+\nu)$ is for the plane stress problem, $\kappa=3-4 \nu$ is for the plane strain problem, and $\nu$ is the Poisson's ratio. In Eq. (1), we denote $\Phi(z)=\phi^{\prime}(z), \Psi(z)=\psi^{\prime}(z)$.

Except for the physical quantities mentioned above, from Eq. (2) a derivative in specified direction (abbreviated as DISD) is introduced [Savruk (1981); Chen (2003)] as follows:

$J_{1}\left(z, \bar{z}, \frac{d \bar{z}}{d z}\right)=\frac{d}{d z}\{-Y+i X\}=\Phi(z)+\overline{\Phi(z)}+\frac{d \bar{z}}{d z}\left(z \overline{\Phi^{\prime}(z)}+\overline{\Psi(z)}\right)=N+i T$.

It is easy to verify that $J_{1}=N+i T$ denotes the normal and tangential tractions along the segment $\overline{z, z+d z}$ (Fig. 1). Second, $J_{1}$ value depends not only on the position of a point " $z$ ", but also on the direction of the segment " $d \bar{z} / d z$ ".

An appropriate complex potential for the problem is [Chen (2003); Chen and Lin (2005)] as follows:

$$
\begin{aligned}
& \phi(z)=\frac{1}{2 \pi} \int_{L} \frac{g(t) d t}{t-z} \\
& \psi(z)=\frac{1}{2 \pi} \int_{L} \frac{g(t) d \bar{t}}{t-z}+\frac{1}{2 \pi} \int_{L} \frac{\overline{g(t)} d t}{t-z}-\frac{1}{2 \pi} \int_{L} \frac{\bar{t} g(t) d t}{(t-z)^{2}},
\end{aligned}
$$

where " $L$ " denotes the curve shown in Fig. 1.

Simply taking the following steps: (a) substituting Eq. (5) into (3), (b) letting the moving point " $z$ " approach to the points $t_{0}^{+}$and $t_{0}^{-}$, which are located on the 


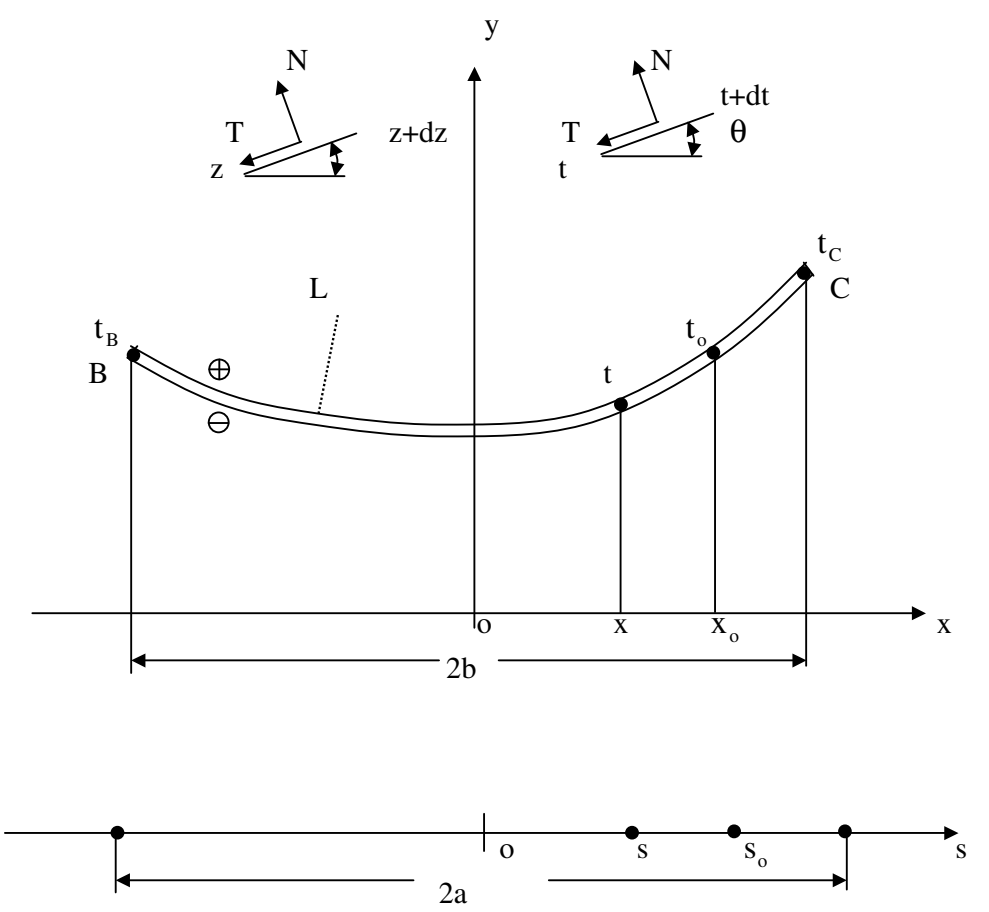

Fig. 1. The curve crack configuration and the curve length coordinate.

upper and lower sides along the curved crack, (c) using the generalized Plemelj formula [Savruk (1981); Chen $(2003)]$, (d) rewriting " $t_{0}$ " as " $t$ ", the following result is obtained:

$g(t)=\frac{2 G}{\kappa+1}\left[\left(u_{y}(t)-i u_{x}(t)\right)^{+}-\left(u_{y}(t)-i u_{x}(t)\right)^{-}\right]=U_{y}(t)-i U_{x}(t), \quad(t \in L)$,

where $\left(u_{y}(t)-i u_{x}(t)\right)^{+}\left(\left(u_{y}(t)-i u_{x}(t)\right)^{-}\right)$denotes the displacements at a point " $t$ " of the upper (lower) face of crack " $L$ ", and $U_{y}(t)-i U_{x}(t)$ denotes the jump value of the displacements, or the COD for the curved crack (Fig. 1). From general analysis in the crack problem, the COD function possesses the following properties [Chen (2003)]:

$$
\begin{aligned}
& g(t)=O\left[\left(t-t_{\mathrm{B}}\right)^{1 / 2}\right], \quad\left(\text { at the vicinity of the left crack tip } t_{\mathrm{B}}\right) . \\
& g(t)=O\left[\left(t-t_{\mathrm{C}}\right)^{1 / 2}\right], \quad\left(\text { at the vicinity of the right crack tip } t_{\mathrm{C}}\right) .
\end{aligned}
$$

In order to obtain the HSIE for the curved crack, one should take the following steps:

(a) After substituting Eq. (5) into (2), the resultant force function $f=-Y+i X$ for any given point " $z$ " can be expressed in the form of an integral, where the unknown function $g(t)$ is involved. 
(b) The value of $N+i T$ along the segment $\overline{z, z+d z}$ is evaluated by using Eq. (4).

(c) Let the moving point " $z$ " approach to the points $t_{0}^{+}$and $t_{0}^{-}$, which are located on the upper and lower side along the curved crack (Fig. 1).

After using the mentioned steps, the HSIE for a curved crack is obtained as follows:

$$
\begin{aligned}
& \frac{1}{\pi} \text { f.p. } \int_{L} \frac{g(t) d t}{\left(t-t_{0}\right)^{2}}+\frac{1}{2 \pi} \int_{L} M_{1}\left(t, t_{0}\right) g(t) d t+\frac{1}{2 \pi} \int_{L} M_{2}\left(t, t_{0}\right) \overline{g(t)} d t \\
& =N\left(t_{0}\right)+i T\left(t_{0}\right), \quad\left(t_{0} \in L\right),
\end{aligned}
$$

where " $L$ " denotes the curve crack configuration, and $N\left(t_{0}\right)+i T\left(t_{0}\right)$ is the traction applied on the crack face, which is given beforehand in the problem (Fig. 1). In Eq. (8), two regular kernels have the following expression:

$$
\begin{aligned}
& M_{1}\left(t, t_{0}\right)=-\frac{d}{d t_{0}}\left\{\frac{d}{d t}\left\{\ln \frac{t-t_{0}}{\bar{t}-\bar{t}_{0}}\right\}\right\}=-\frac{1}{\left(t-t_{0}\right)^{2}}+\frac{1}{\left(\bar{t}-\bar{t}_{0}\right)^{2}} \frac{d \bar{t}}{d t} \frac{d \bar{t}_{0}}{d t_{0}}, \\
& M_{2}\left(t, t_{0}\right)=\frac{d}{d t_{0}}\left\{\frac{d}{d t}\left\{\frac{t-t_{0}}{\bar{t}-\bar{t}_{0}}\right\}\right\}=\frac{1}{\left(\bar{t}-\bar{t}_{0}\right)^{2}}\left(\frac{d \bar{t}}{d t}+\frac{d \bar{t}_{0}}{d t_{0}}\right)-\frac{2\left(t-t_{0}\right)}{\left(\bar{t}-\bar{t}_{0}\right)^{3}} \frac{d \bar{t}}{d t} \frac{d \bar{t}_{0}}{d t_{0}} .
\end{aligned}
$$

In Eq. (8), the first integral with a notation "f.p." is a hypersingular integral, which should be defined in the sense of finite part integral proposed by Hadamard [1923].

Once a solution for Eq. (8) is obtained, the SIF at the left crack tip B (Fig. 1) can be evaluated by [Savruk (1981); Chen (2003)]

$$
\left(K_{1}-i K_{2}\right)_{\mathrm{B}}=\sqrt{2 \pi} \operatorname{Lim}_{t \rightarrow t_{\mathrm{B}}} \sqrt{\left|t-t_{\mathrm{B}}\right|} g^{\prime}(t), \quad\left(g^{\prime}(t)=d g(t) / d t\right) .
$$

Similarly, for the right crack tip $\mathrm{C}$ we have

$$
\left(K_{1}-i K_{2}\right)_{\mathrm{C}}=-\sqrt{2 \pi} \operatorname{Lim}_{t \rightarrow t_{\mathrm{C}}} \sqrt{\left|t-t_{\mathrm{C}}\right|} g^{\prime}(t) .
$$

The curve length coordinate technique is used to solve the HSIE numerically [Mayrhofer et al. (1992); Chen (2003)]. Some quadrature rules are introduced in the Appendix. If the COD is not overlapped, the above-mentioned analysis provides a solution for the curved crack problem.

Since the displacement is overlapped in the contact problem of an arc crack, necessary modifications must be taken to the solution of the contact problem. Without losing generality, we will take the arc crack under the remote traction $\sigma_{x y}^{\infty}=p$ as an example (Fig. 2(a)). It is shown by computation that the left portion of the arc crack is under contact. The original problem is considered as a superposition of the uniform field and the perturbation field, which are shown in Figs. 2(b) and 2(c), respectively.

After using some quadrature rule (see Appendix), Eq. (8) may be reduced to

$$
\mathbf{K U}=\mathbf{P},
$$




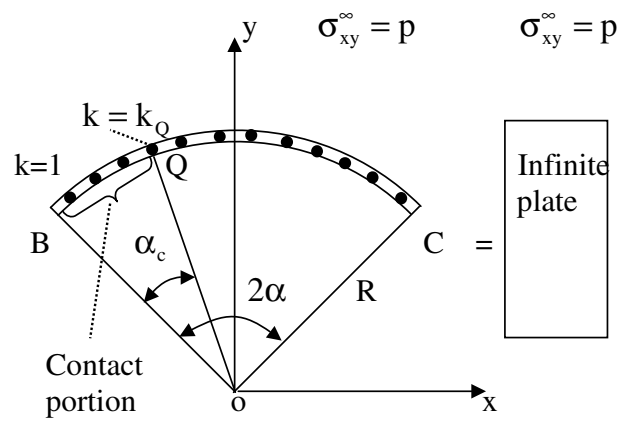

(a)

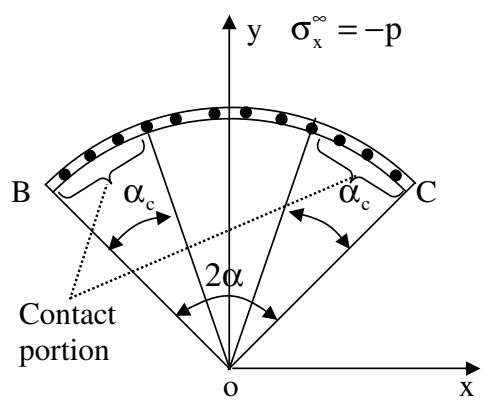

(d)

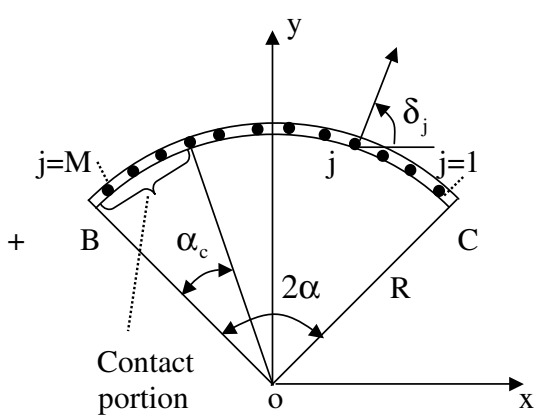

(c)

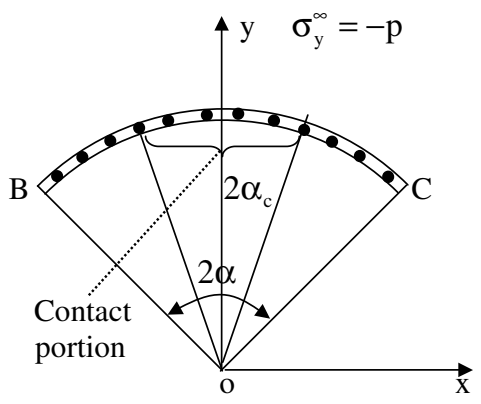

(e)

Fig. 2. (a) The original problem for an arc crack under remote traction $\sigma_{x y}^{\infty}=p$, (b) the uniform stress field, (c) the perturbation field, (d) the original problem for an arc crack under remote loading $\sigma_{x}^{\infty}=-p$, (e) the original problem for an arc crack under remote loading $\sigma_{y}^{\infty}=-p$.

where " $K$ " $(2 M \times 2 M)$ is a matrix resulted from Eq. (8), " $M$ " is the number of the collocation points in the qudrature rule (Fig. 2(c)), and

$$
\begin{aligned}
& \mathbf{U}=\left(U_{y, 1}-U_{x, 1}, \ldots, U_{y, j}-U_{x, j}, \ldots, U_{y, M}-U_{x, M}\right)^{\mathrm{T}}, \quad(2 M \times 1), \\
& \mathbf{P}=\left(N_{1} T_{1}, \ldots, N_{j} T_{j}, \ldots, N_{M} T_{M}\right)^{\mathrm{T}}, \quad(2 M \times 1) .
\end{aligned}
$$

In Eqs. (13) and (14), $U_{y, j}$ and $-U_{x, j}$ denote the COD defined by Eq. (6) at the $j$ th node, and $N_{j}$ and $T_{j}$ denote the normal and tangential tractions at the $j$ th node (Fig. 2(c)).

It is easy to see that the form of Eq. (12) is not appropriate to solve the contact crack problem. In the following we define

$$
\frac{2 G}{\kappa+1}\left[\left(u_{n}(t)+i u_{t}(t)\right)^{+}-\left(u_{n}(t)+i u_{t}(t)\right)^{-}\right]=U_{n}(t)+i U_{t}(t), \quad(t \in L),
$$

where $\left(u_{n}(t)+i u_{t}(t)\right)^{+}\left(\left(u_{n}(t)+i u_{t}(t)\right)^{-}\right)$denotes the normal and tangential displacements at a point " $t$ " of the upper (lower) face of crack " $L$ ", and $U_{n}(t)+i U_{t}(t)$ 
denotes the jump value of the displacements, or the COD for the curved crack (Fig. 2(c)).

From Eqs. (6) and (15), we will find the following relation:

$$
U_{n}=U_{x} \cos \delta+U_{y} \sin \delta, \quad U_{t}=-U_{x} \sin \delta+U_{y} \cos \delta,
$$

where $\delta$ stands for an inclination angle of normal at a point on the arc (Fig. 2(c)).

By using this relation (16), Eq. (12) is reduced to

$$
\mathbf{L} \mathbf{U}_{\mathbf{R}}=\mathbf{P},
$$

where " $L "(2 M \times 2 M)$ is a matrix resulted from the relation (16), and

$$
\mathbf{U}_{\mathbf{R}}=\left(U_{n, 1} U_{t, 1}, \ldots, U_{n, j} U_{t, j}, \ldots, U_{n, M} U_{t, M}\right)^{T}, \quad(2 M \times 1) .
$$

Clearly, the elements in the matrix " $L$ " are defined by

$$
\begin{aligned}
L_{i, 2 j-1} & =-K_{i, 2 j} \cos \delta_{j}+K_{i, 2 j-1} \sin \delta_{j}, \\
L_{i, 2 j} & =K_{i, 2 j} \sin \delta_{j}+K_{i, 2 j-1} \cos \delta_{j}, \quad(i=1,2, \ldots, 2 M),
\end{aligned}
$$

where, for example, $K_{i, 2 j-1}$ and $K_{i, 2 j}$ are elements in the matrix $K$ shown in Eq. (12), and $\delta_{j}$ stands for an inclined angle of normal at the $j$ th node on the arc (Fig. 2(c)).

The detailed computation is composed of the following steps:

(a) Assume that the left portion of the arc crack is contacted. Alternatively speaking, the $U_{n, k}$ components are vanishing along the nodes from $k=1, k=2, \ldots$, to $k=k_{Q}$, where the " $k_{Q}$ " is a temporary assumed value (Fig. $2(\mathrm{a})$ ).

(b) Under the condition expressed in step (a), we solve the boundary value problem shown in Fig. 2(c). To this end, we need to use Eq. (17), and make some modification for the matrix $\mathbf{L}$. In the case of letting the $U_{n, k}$ components vanish along the nodes from $k=1, k=2, \ldots$ to $k=k_{Q}$, we simply let relevant elements in the matrix be a huge value, or $L(2 k-1,2 k-1)=10^{20}$, for $k=1, k=2, \ldots$ to $k=k_{Q}$. Since the matrix $\mathbf{L}$ has been modified, the algebraic equation (17) now is rewritten in the form

$$
\mathbf{L}_{\mathbf{m d}} \mathbf{U}_{\mathbf{R}}=\mathbf{P}
$$

where the matrix $\mathbf{L}_{\mathbf{m d}}$ is a modified matrix obtained from $\mathbf{L}$.

Therefore, from Eq. (17a), we can get solution for (a) $U_{n, k}=0$ for $k=1, k=$ $2, \ldots$ to $k=k_{Q}$ (this is actually obtained from the huge value correction for some elements in the matrix $\mathbf{L}$ ), (b) $U_{t, k}$ for $k=1, k=2, \ldots$ to $k=k_{Q}$ and (c) $U_{n, k}$ and $U_{t, k}$ for $k=k_{Q}+1, k=k_{Q}+2, \ldots$ to $k=M$. In conclusion, the vector " $\mathbf{U}_{\mathbf{R}}$ " can be obtained from the solution of Eq. (17a).

(c) Physically, Eq. (17) means that there is a definite traction applied along the crack face when the COD vector $\mathbf{U}_{\mathbf{R}}$ is assumed, and this relation is expressed as $\mathbf{P}=\mathbf{L}_{\mathbf{R}}$. Once the vector " $\mathbf{U}_{\mathbf{R}}$ " is obtained from the solution of Eq. (17a), the traction along the crack face including the contacted portion can be evaluated immediately by using Eq. (17) (or $\mathbf{P}=\mathbf{L}_{\mathbf{R}}$ ) for the problem shown by Fig. 2(c). 
(d) By using the superposition principle, the contact stress $N_{Q}\left(=\sigma_{n, Q}\right)$ at the node $k=k_{Q}$ (or at the node point " $Q$ "), in the problem shown by Fig. 2(a), is obtainable.

A condition for determining the boundary of the contact is introduced

$$
N_{Q}=0, \quad \text { or } \quad \sigma_{n, Q}=0, \quad\left(\text { at the node } k=k_{Q}\right) .
$$

Since the computation in the present study is discrete, $k_{Q}$ may be determined by the following rule. When $k_{Q}$ value is increasing gradually (for example, let $k_{Q}=$ $58,59,60, \ldots)$, the first one that satisfies the condition $N_{Q}>0$ and $N_{Q} \approx 0$ is the suitable one for the investigated $k_{Q}$ value. Therefore, the contact curve range "BQ" is determined numerically (Fig. 2(a)).

\section{Numerical Examples}

Three numerical examples with different remote stresses are carried out below.

\subsection{An example with the remote shear stress $\sigma_{x y}^{\infty}=p$}

In the first example, the loading is the remote shear stress $\sigma_{x y}^{\infty}=p$ (Fig. 2(a)). In computation, we choose the number of the collocation points $M=155$. The calculated results for SIFs at the crack tips B and $\mathrm{C}$ are expressed as

$$
\begin{aligned}
& K_{1 \mathrm{~B}}=F_{1 \mathrm{~B}}(\alpha) p \sqrt{\pi c}, \quad K_{1 \mathrm{C}}=F_{1 \mathrm{C}}(\alpha) p \sqrt{\pi c}, \\
& K_{2 \mathrm{~B}}=F_{2 \mathrm{~B}}(\alpha) p \sqrt{\pi c}, \quad K_{2 \mathrm{C}}=F_{2 \mathrm{C}}(\alpha) p \sqrt{\pi c}, \quad \text { where } \sigma_{x y}^{\infty}=p, c=R \sin \alpha .
\end{aligned}
$$

In addition, the contact angle $\alpha_{c}$ is expressed by (Fig. 2(a))

$$
\alpha_{\mathrm{c}}=H(\alpha) \alpha .
$$

In addition, in the case of not considering the contact effect, the available results [Cotterell and Rice (1980)] are as follows:

$$
\begin{aligned}
& K_{1 \mathrm{~B}}=-K_{1 \mathrm{C}}=G_{1 \mathrm{~B}}(\alpha) p \sqrt{\pi c}, \\
& K_{2 \mathrm{~B}}=K_{2 \mathrm{C}}=G_{2 \mathrm{~B}}(\alpha) p \sqrt{\pi c}, \quad \text { where } \sigma_{x y}^{\infty}=p, c=R \sin \alpha,
\end{aligned}
$$

where

$$
\begin{aligned}
& G_{1 \mathrm{~B}}(\alpha)=-\left(\sin (3 \alpha / 2)+\sin ^{3}(\alpha / 2)\right), \\
& G_{2 \mathrm{~B}}(\alpha)=\cos (3 \alpha / 2)+\cos (\alpha / 2) \sin ^{2}(\alpha / 2) .
\end{aligned}
$$

The obtained results for $F_{1 \mathrm{~B}}(\alpha), F_{2 \mathrm{~B}}(\alpha), F_{1 \mathrm{C}}(\alpha), F_{2 \mathrm{C}}(\alpha), H(\alpha), G_{1 \mathrm{~B}}(\alpha)$, and $G_{2 \mathrm{~B}}(\alpha)$ are tabulated in Table 1 and shown in Fig. 3.

The normal COD $\left(U_{n}\right)$ is vanishing along the contact curve range "BQ" in the derivation, therefore, $K_{1 \mathrm{~B}}=0$ ( or $F_{1 \mathrm{~B}}=0$ ). The listed $F_{1 \mathrm{~B}}$ values in Table 1 are from the numerical solution, and they are varying within the range -0.0017 to 0.0019 , a rather small value. This can partly prove the rightness of the suggested formulation. 
Table 1. Non-dimensional SIFs $F_{1 \mathrm{~B}}(\alpha), F_{1 \mathrm{C}}(\alpha), F_{2 \mathrm{~B}}(\alpha), F_{2 \mathrm{C}}(\alpha), G_{1 \mathrm{~B}}(\alpha), G_{1 \mathrm{C}}(\alpha), G_{2 \mathrm{~B}}(\alpha)$, $G_{2 \mathrm{C}}(\alpha)$, and $H(\alpha)$ value for an arc crack under contact with loading $\sigma_{x y}^{\infty}=p$ (see Fig. 2(a) and Eqs. (21)-(24)).

\begin{tabular}{|c|c|c|c|c|c|c|c|c|c|}
\hline & \multicolumn{9}{|c|}{$\alpha$ (degree) } \\
\hline & 10 & 20 & 30 & 40 & 50 & 60 & 70 & 80 & 90 \\
\hline \multicolumn{10}{|c|}{ Considering the contact effect } \\
\hline$F_{1 \mathrm{~B}}$ & 0.0003 & -0.0009 & 0.0015 & -0.0019 & 0.0019 & -0.0017 & 0.0014 & -0.0011 & 0.0009 \\
\hline$F_{1 \mathrm{C}}$ & 0.2812 & 0.5412 & 0.7573 & 0.9171 & 1.0015 & 1.0075 & 0.9280 & 0.7673 & 0.5322 \\
\hline$F_{2 \mathrm{~B}}$ & 0.9801 & 0.9214 & 0.8278 & 0.7013 & 0.5550 & 0.3873 & 0.2207 & 0.0501 & -0.0970 \\
\hline$F_{2 \mathrm{C}}$ & 0.9667 & 0.8693 & 0.7158 & 0.5151 & 0.2872 & 0.0395 & -0.1996 & -0.4257 & -0.6101 \\
\hline $\mathrm{H}$ & 0.6758 & 0.6948 & 0.7139 & 0.7331 & 0.7525 & 0.7719 & 0.7914 & 0.8110 & 0.8307 \\
\hline \multicolumn{10}{|c|}{ Not considering the contact effect } \\
\hline$G_{1 \mathrm{~B}}$ & -0.2595 & -0.5052 & -0.7244 & -0.9060 & -1.0414 & -1.1250 & -1.1546 & -1.1316 & -1.0607 \\
\hline$G_{1 \mathrm{C}}$ & 0.2595 & 0.5052 & 0.7244 & 0.9060 & 1.0414 & 1.1250 & 1.1546 & 1.1316 & 1.0607 \\
\hline$G_{2 \mathrm{~B}}$ & 0.9735 & 0.8957 & 0.7718 & 0.6099 & 0.4207 & 0.2165 & 0.0107 & -0.1835 & -0.3535 \\
\hline$G_{2 \mathrm{C}}$ & 0.9735 & 0.8957 & 0.7718 & 0.6099 & 0.4207 & 0.2165 & 0.0107 & -0.1835 & -0.3535 \\
\hline
\end{tabular}

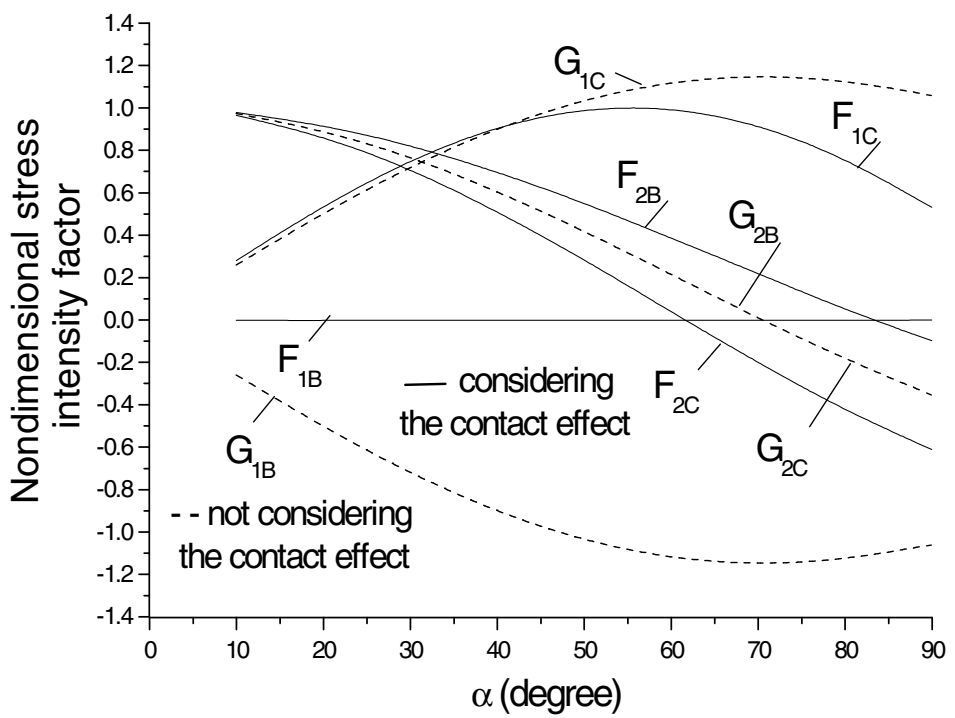

Fig. 3. Non-dimensional SIFs $F_{1 \mathrm{~B}}(\alpha), F_{1 \mathrm{C}}(\alpha), F_{2 \mathrm{~B}}(\alpha), F_{2 \mathrm{C}}(\alpha), G_{1 \mathrm{~B}}(\alpha), G_{1 \mathrm{C}}(\alpha), G_{2 \mathrm{~B}}(\alpha), G_{2 \mathrm{C}}(\alpha)$, and $H(\alpha)$ value for an arc crack under contact with loading $\sigma_{x y}^{\infty}=p$ (see Fig. 2(a) and Eqs. (21)-(24)).

It is seen from the tabulated results that, in the case of considering contact effect, we have $F_{1 \mathrm{~B}} \approx 0$. However, in the case of not considering contact effect, the relevant $G_{1 \mathrm{~B}}$ values change from $G_{1 \mathrm{~B}}=-0.2595$ (for $\alpha=10^{\circ}$ ) to $G_{1 \mathrm{~B}}=-1.0607$ (for $\alpha=90^{\circ}$ ). A negative first mode SIF (or $G_{1 \mathrm{~B}}<0$ ), or the overlapping of the normal COD, is not acceptable from the physical situation of deformation. 
For a small value of $\alpha$, the following results from two conditions are comparable. For example, if $\alpha=10^{\circ}$, we have $F_{1 \mathrm{C}}=0.2812, F_{2 \mathrm{~B}}=0.9801, F_{2 \mathrm{C}}=0.9667$, and $G_{1 \mathrm{C}}=0.2595, G_{2 \mathrm{~B}}=0.9735, G_{2 \mathrm{C}}=0.9735$.

The ratios of the contact portion $H(\alpha)\left(=\alpha_{c} / \alpha\right)$ are varying within the range from 0.6758 to 0.8307 .

\subsection{An example with the remote shear stress $\sigma_{x}^{\infty}=-p$}

In the second example, the loading is the remote shear stress $\sigma_{x}^{\infty}=-p$ (Fig. $\left.2(\mathrm{~d})\right)$. In computation, we choose the number of the collocation points $M=155$. The calculated results for SIFs at the crack tips B and $\mathrm{C}$ are expressed as

$$
\begin{aligned}
& K_{1 \mathrm{~B}}=K_{1 \mathrm{C}}=F_{1 \mathrm{~B}}(\alpha) p \sqrt{\pi c}, \\
& K_{2 \mathrm{~B}}=-K_{2 \mathrm{C}}=F_{2 \mathrm{~B}}(\alpha) p \sqrt{\pi c}, \quad \text { where } \sigma_{x}^{\infty}=-p, c=R \sin \alpha .
\end{aligned}
$$

In addition, the contact angle $\alpha_{c}$ is expressed by

$$
\alpha_{c}=H(\alpha) \alpha .
$$

In addition, in the case of not considering the contact effect, the available results [Cotterell and Rice (1980)] are as follows:

$$
\begin{aligned}
& K_{1 \mathrm{~B}}=K_{1 \mathrm{C}}=G_{1 \mathrm{~B}}(\alpha) p \sqrt{\pi c}, \\
& K_{2 \mathrm{~B}}=-K_{2 \mathrm{C}}=G_{2 \mathrm{~B}}(\alpha) p \sqrt{\pi c}, \quad \text { where } \sigma_{x}^{\infty}=-p, c=R \sin \alpha,
\end{aligned}
$$

where

$$
\begin{aligned}
& G_{1 \mathrm{~B}}(\alpha)=\frac{1}{2}\left[-\left(1+\sin ^{2}(\alpha / 2) \cos ^{2}(\alpha / 2)\right) \frac{\cos (\alpha / 2)}{1+\sin ^{2}(\alpha / 2)}+\cos (3 \alpha / 2)\right], \\
& G_{2 \mathrm{~B}}(\alpha)=\frac{1}{2}\left[-\left(1+\sin ^{2}(\alpha / 2) \cos ^{2}(\alpha / 2)\right) \frac{\sin (\alpha / 2)}{1+\sin ^{2}(\alpha / 2)}+\sin (3 \alpha / 2)\right] .
\end{aligned}
$$

\begin{tabular}{|c|c|c|c|c|c|c|c|c|c|}
\hline & \multicolumn{9}{|c|}{$\alpha($ degree $)$} \\
\hline & 10 & 20 & 30 & 40 & 50 & 60 & 70 & 80 & 90 \\
\hline \multicolumn{10}{|c|}{ Considering the contact effect } \\
\hline$F_{1 \mathrm{~B}}$ & 0.0000 & -0.0001 & -0.0001 & 0.0002 & -0.0004 & 0.0004 & 0.0011 & -0.0008 & -0.0014 \\
\hline$F_{2 \mathrm{~B}}$ & 0.0863 & 0.1666 & 0.2354 & 0.2877 & 0.3191 & 0.3269 & 0.3096 & 0.2668 & 0.2012 \\
\hline $\mathrm{H}$ & 0.4434 & 0.4434 & 0.4434 & 0.4601 & 0.4771 & 0.4942 & 0.5292 & 0.5469 & 0.5829 \\
\hline \multicolumn{10}{|c|}{ Not considering the contact effect } \\
\hline$G_{1 \mathrm{~B}}$ & -0.0151 & -0.0590 & -0.1274 & -0.2141 & -0.3115 & -0.4114 & -0.5056 & -0.5867 & -0.6482 \\
\hline$G_{2 \mathrm{~B}}$ & 0.0858 & 0.1633 & 0.2247 & 0.2641 & 0.2774 & 0.2625 & 0.2195 & 0.1504 & 0.0589 \\
\hline
\end{tabular}

The obtained results for $F_{1 \mathrm{~B}}(\alpha), F_{2 \mathrm{~B}}(\alpha), H(\alpha), G_{1 \mathrm{~B}}(\alpha)$, and $G_{2 \mathrm{~B}}(\alpha)$ are tabulated in Table 2 and shown in Fig. 4.

Table 2. Non-dimensional SIFs $F_{1 \mathrm{~B}}(\alpha), F_{2 \mathrm{~B}}(\alpha), G_{1 \mathrm{~B}}(\alpha), G_{2 \mathrm{~B}}(\alpha)$, and $H(\alpha)$ value for an arc crack under contact with loading $\sigma_{x}^{\infty}=-p$ (see Fig. 2(d) and Eqs. (25)-(28)). 


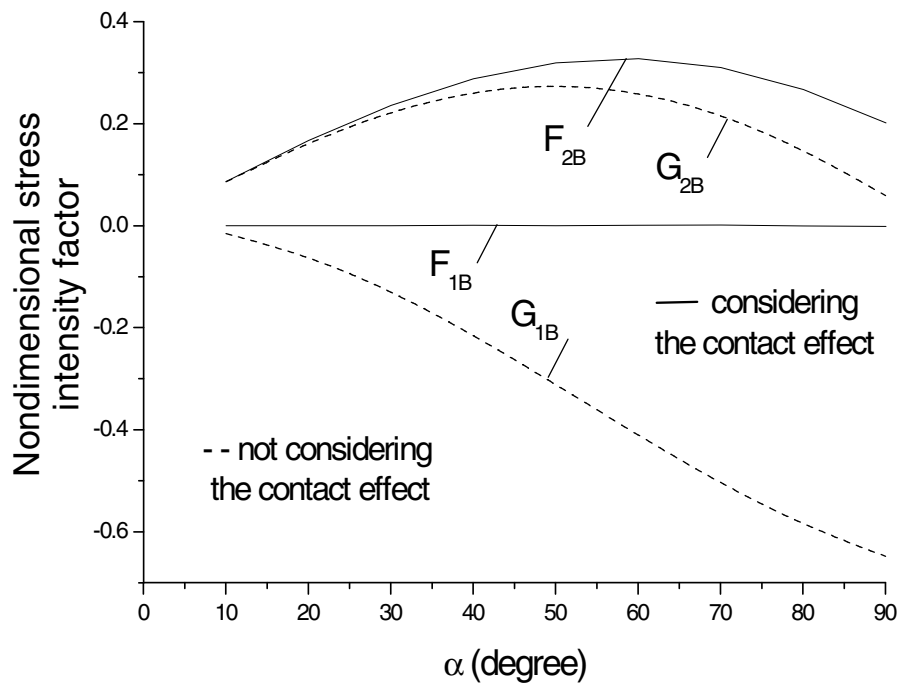

Fig. 4. Non-dimensional SIFs $F_{1 \mathrm{~B}}(\alpha), F_{2 \mathrm{~B}}(\alpha), G_{1 \mathrm{~B}}(\alpha), G_{2 \mathrm{~B}}(\alpha)$, and $H(\alpha)$ value for an arc crack under contact with loading $\sigma_{x}^{\infty}=-p$ (see Fig. 2(d) and Eqs. (25)-(28)).

It is seen from the tabulated results that, in the case of considering contact effect, we have $F_{1 \mathrm{~B}} \approx 0$. However, in the case of not considering contact effect, the relevant $G_{1 \mathrm{~B}}$ values change from $G_{1 \mathrm{~B}}=-0.0151$ (for $\alpha=10^{\circ}$ ) to $G_{1 \mathrm{~B}}=-0.6482$ (for $\alpha=90^{\circ}$ ). As before, a negative first mode SIF (or $G_{1 \mathrm{~B}}<0$ ), or the overlapping of the normal COD, is not acceptable from the physical situation of deformation. We see from Fig. 4 that the computed $F_{2 \mathrm{~B}}$ and $G_{2 \mathrm{~B}}$ values are comparable. The ratios of the contact portion $H(\alpha)\left(=\alpha_{c} / \alpha\right)$ are varying within the range from 0.4434 to 0.5829 .

\subsection{An example with the remote shear stress $\sigma_{y}^{\infty}=-p$}

In the third example, the loading is the remote shear stress $\sigma_{y}^{\infty}=-p$ (Fig. 2(e)). In computation, we choose the number of the collocation points $M=155$. The calculated results for SIFs at the crack tips B and C are expressed as

$$
\begin{aligned}
& K_{1 \mathrm{~B}}=K_{1 \mathrm{C}}=F_{1 \mathrm{~B}}(\alpha) p \sqrt{\pi c}, \\
& K_{2 \mathrm{~B}}=-K_{2 \mathrm{C}}=F_{2 \mathrm{~B}}(\alpha) p \sqrt{\pi c}, \quad \text { where } \sigma_{y}^{\infty}=-p, c=R \sin \alpha .
\end{aligned}
$$

In addition, the contact angle $\alpha_{\mathrm{c}}$ is expressed by

$$
\alpha_{\mathrm{c}}=H(\alpha) \alpha \text {. }
$$

In addition, in the case of not considering the contact effect, the available results [Cotterell et al. (1980)] are as follows:

$$
\begin{aligned}
& K_{1 \mathrm{~B}}=K_{1 \mathrm{C}}=G_{1 \mathrm{~B}}(\alpha) p \sqrt{\pi c} \\
& K_{2 \mathrm{~B}}=-K_{2 \mathrm{C}}=G_{2 \mathrm{~B}}(\alpha) p \sqrt{\pi c}, \quad \text { where } \sigma_{y}^{\infty}=-p, c=R \sin \alpha .
\end{aligned}
$$


where

$$
\begin{aligned}
& G_{1 \mathrm{~B}}(\alpha)=\frac{1}{2}\left[-\left(1-\sin ^{2}(\alpha / 2) \cos ^{2}(\alpha / 2)\right) \frac{\cos (\alpha / 2)}{1+\sin ^{2}(\alpha / 2)}-\cos (3 \alpha / 2)\right], \\
& G_{2 \mathrm{~B}}(\alpha)=\frac{1}{2}\left[-\left(1-\sin ^{2}(\alpha / 2) \cos ^{2}(\alpha / 2)\right) \frac{\sin (\alpha / 2)}{1+\sin ^{2}(\alpha / 2)}-\sin (3 \alpha / 2)\right] .
\end{aligned}
$$

The obtained results for $F_{1 \mathrm{~B}}(\alpha), F_{2 \mathrm{~B}}(\alpha), H(\alpha), G_{1 \mathrm{~B}}(\alpha)$, and $G_{2 \mathrm{~B}}(\alpha)$ are tabulated in Table 3 and shown in Fig. 5 .

Table 3. Non-dimensional SIFs $F_{1 \mathrm{~B}}(\alpha), F_{2 \mathrm{~B}}(\alpha), G_{1 \mathrm{~B}}(\alpha), G_{2 \mathrm{~B}}(\alpha)$, and $H(\alpha)$ value for an arc crack under contact with loading $\sigma_{y}^{\infty}=-p$ (see Fig. 2(e) and Eqs. (29)-(32)).

\begin{tabular}{lrrrrrrrrr}
\hline & \multicolumn{1}{c}{$\alpha$ (degree) } \\
\cline { 2 - 9 } & 10 & 20 & 40 & 50 & 60 & 70 & 80 & \multicolumn{1}{c}{90} \\
\hline Considering the contact effect \\
$F_{1 \mathrm{~B}}$ & 0.0000 & 0.0000 & 0.0000 & 0.0000 & 0.0000 & 0.0000 & 0.0113 & 0.0444 & 0.0715 \\
$F_{2 \mathrm{~B}}$ & -0.0862 & -0.1658 & -0.2328 & -0.2820 & -0.3094 & -0.3123 & -0.2902 & -0.2433 & -0.1727 \\
$H$ & 1.0000 & 1.0000 & 1.0000 & 1.0000 & 1.0000 & 1.0000 & 0.9286 & 0.8137 & 0.7246 \\
Not considering the contact effect & & & & & & \\
$G_{1 \mathrm{~B}}$ & -0.9736 & -0.8970 & -0.7779 & -0.6272 & -0.4575 & -0.2815 & -0.1107 & 0.0447 & 0.1768 \\
$G_{2 \mathrm{~B}}$ & -0.1723 & -0.3318 & -0.4673 & -0.5703 & -0.6360 & -0.6625 & -0.6511 & -0.6053 & -0.5303 \\
\hline
\end{tabular}

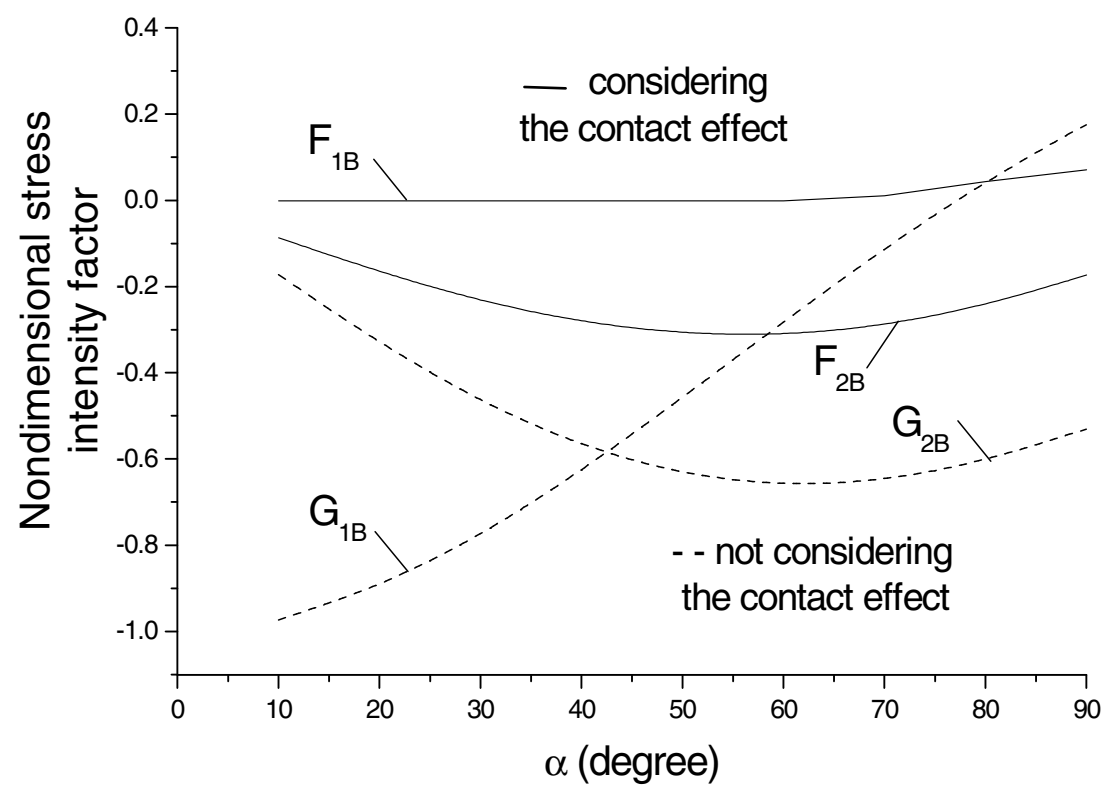

Fig. 5. Non-dimensional SIFs $F_{1 \mathrm{~B}}(\alpha), F_{2 \mathrm{~B}}(\alpha), G_{1 \mathrm{~B}}(\alpha), G_{2 \mathrm{~B}}(\alpha)$, and $H(\alpha)$ value for an arc crack under contact with loading $\sigma_{y}^{\infty}=-p$ (see Fig. 2(e) and Eqs. (29)-(32)). 
Some particular features can be found from the present example. If $\alpha \leq 60^{\circ}$, the full contact takes place (or $\alpha_{c} / \alpha=1$ ), and $F_{1 \mathrm{~B}}=0$. However, if $\alpha \geq 70^{\circ}$, the incomplete contact takes place, or $\alpha_{c} / \alpha<1$. Alternatively speaking, in this case contact does not take place at the vicinity of the crack tip and $F_{1 \mathrm{~B}} \neq 0$. This feature is not the same as in the previous examples.

\section{Conclusions}

As mentioned previously, if one does not consider the contact effect for a contact arc crack, the obtained solution for the SIFs is of no sense. This paper provides an exact formulation and an accurate numerical result for the contact arc crack. A concrete example is introduced below. For an arc crack with remote loading $\sigma_{x y}^{\infty}=p$ and the span angle $2 \alpha=100^{\circ}$, we have the non-dimensional SIFs $F_{1 \mathrm{~B}} \approx 0, F_{2 \mathrm{~B}}=0.5550$ (for the left crack tip B) and $F_{1 \mathrm{C}}=1.0015, F_{2 \mathrm{C}}=0.2872$ (for the right crack tip C). Therefore, it can be concluded that failure must be initiated at the right crack tip C (Fig. 2(a)).

As mentioned previously, the HSIE is an effective tool for the solution of the contact crack problem since the COD can be obtained from the solution.

The above-mentioned procedure may be used to the contact problem of arbitrary configuration of the curved crack. In the first step, we may complete a computation under full contact condition, or all $U_{n}$ values on nodes are equal to zero. Further, from the numerical solution one may find on some nodes, for example, the normal traction $N_{j}<0$ (for $j=1,2, \ldots, j_{\mathrm{c}}$ ) and $N_{j}>0$ (for $\left.j=j_{\mathrm{c}}+1, \ldots, M\right)$. In this case, one may make another computation under the condition $U_{n}=0$ on the nodes $j=1,2, \ldots, j_{\mathrm{c}}$. In fact, the solution for contact crack problem must depend on the iteration. Once the contact condition (similar to the condition shown by Eq. (20)) at the margin nodes of contact portion is satisfied, the final result is obtained.

\section{Appendix}

\section{Numerical solution of the hypersingular integral equation}

The curve length coordinate technique is suggested to solve the HSIE numerically [Mayrhofer and Fischer (1992); Chen (2003)]. In the technique, the curve configuration is mapped on a real axis " $s$ " with an interval with length "2a" (Fig. 1). Clearly, this is one-to-one mapping. Alternatively speaking, for a point on the curved crack, there has a mapping point on the real axis " $s$ ", and the inverse is true.

The mapping relation is expressed by the function $t(s)$. After using the mentioned mapping, function $g(t)$ is rewritten in the form

$$
\left.g(t)\right|_{t=t(s)}=h(s)=\sqrt{a^{2}-s^{2}} H(s),
$$

where $H(s)=H_{1}(s)+i H_{2}(s)$ is a regular function. 
After using the substitution (A.1), the following integration rules are sufficient to solve the HSIE. The integration rule for a hypersingular integral with the integrand $\sqrt{a^{2}-s^{2}} H(s)$ [Mayrhofer and Fischer (1992)] is as follows:

$$
\frac{1}{\pi} \mathrm{f} . \mathrm{p} \cdot \int_{-a}^{a} \frac{\sqrt{a^{2}-s^{2}} H(s) d s}{\left(s-s_{0}\right)^{2}}=\sum_{j=1}^{M+1} W_{j}\left(s_{0}\right) H\left(s_{j}\right), \quad\left(\left|s_{0}\right|<a\right),
$$

where $H(s)$ is a given regular function, $M$ is an assumed integer, and

$$
\begin{gathered}
s_{j}=a \cos \left(\frac{j \pi}{M+2}\right), \quad(j=1,2, \ldots, M+1) . \\
W_{j}\left(s_{0}\right)=-\frac{2}{M+2} \sum_{n=0}^{M}(n+1) \sin \left(\frac{j \pi}{M+2}\right) \sin \left(\frac{(n+1) j \pi}{M+2}\right) U_{n}\left(\frac{s_{o}}{a}\right), \\
(j=1,2, M+1) . \\
U_{n}(q)=\frac{\sin ((n+1) \theta)}{\sin \theta}, \quad \text { where } \theta=\arccos (q), \quad(|q| \leq 1) .
\end{gathered}
$$

In Eq. (A.4), $U_{n}(q)$ denotes the Chebyshev polynomials of second kind.

For the integrand with regular function the following integration rule is suggested

$$
\frac{1}{\pi} \int_{-a}^{a} \sqrt{a^{2}-s^{2}} H(s) d s=\frac{1}{M+2} \sum_{j=1}^{M+1}\left(a^{2}-s_{j}^{2}\right) H\left(s_{j}\right) .
$$

Second, once the solution for $H\left(s_{j}\right)(j=1,2, \ldots, M+1)$ is obtained from a solution of algebraic equation, function $H(s)$ can be determined by

$$
H(s)=\sum_{n=0}^{M} c_{n} U_{n}\left(\frac{s}{a}\right), \quad|s| \leq a,
$$

where

$$
c_{n}=\frac{2}{M+2} \sum_{j=1}^{M+1} \sin \left(\frac{j \pi}{M+2}\right) \sin \left(\frac{(n+1) j \pi}{M+2}\right) H\left(s_{j}\right) .
$$

Clearly, the values of $H(-a)$ and $H(a)$ can also be evaluated from Eq. (A.7).

\section{References}

Basista, M. and Gross, D. [2000] A note on crack interactions under compression, Int. J. Fract. 102, L67-72.

Beghini, M. and Bertini, L. [1996] Effective stress intensity factor and contact stress for a partially closed Griffith crack in bending, Eng. Fract. Mech. 54, 667-678.

Birinci, A. and Cakiroglu, F. L. [2003] Partial closure of a crack located in an infinite elastic layer, Eur. J. Mech. A Solids 22, 583-590.

Chao, R. and Laws, N. [1992] Closure of an arc crack in an isotropic homogeneous material due to uniaxial loading, Quat. J. Mech. Appl. Math. 45, 629-640. 
Chao, R. and Rau, M. Y. [1995] Frictional contact of an arc crack in an isotropic homogeneous materials due to uniaxial loading, Eng. Fract. Mech. 50, 121-130.

Chen, Y. Z. [1993] Numerical solution of a curved crack problem by using hypersingular integral equation, Eng. Fract. Mech. 46, 275-283.

Chen, Y. Z. [1999] A contact problem in an infinite plate, Int. J. Eng. Sci. 37, 621-630.

Chen, Y. Z. [2004] Numerical solution for multiple crack problem in an infinite plate under compression, Int. J. Fract. 129, 51-62.

Chen, Y. Z. [2003] A numerical solution technique of hypersingular integral equation for curved cracks, Com. Num. Meth. Eng. 19, 645-655.

Chen, Y. Z. and Lin, X. Y. [2005] Numerical solution of hypersingular integral equation for curved cracks in circular regions, Int. J. Fract. 135, 205-222.

Cotterell, B. and Rice J. R. [1980] Slightly curved or kinked cracks, Int. J. Fract. 16, $155-169$.

De Bremaecker, J.-CI. Ferris, M. C. and Ralph, D. [2000a] Compressional fractures considered as contact problems and mixed complimentary problems, Eng. Fract. Mech. 66, 287-303.

De Bremaecker, J.-CI. and Ferris, M. C. [2000b] A comparison of two algorithms for solving closed crack problems, Eng. Fract. Mech. 66, 601-605.

Hadamard, J. [1923] Lectures on Cauchy's Problem in Linear Differential Equations (University Press, Yale).

Helsing, J. and Peters, G. [2000] An efficient numerical algorithm for cracks partly in frictional contact, SIAM J. Appl. Math. 61, 551-566.

Kaya, A. C. and Erdogan, F. [1987] On the solutions of integral equations with strongly singular kernels, Quart. Appl. Mech. 45, 105-122.

Krenk, S. [1975] On the use of the interpolation polynomial for solutions singular of integral equations, Quart. Appl. Math. 32, 479-484.

Lauterbach, B. and Gross, D. [1998] Crack growth in brittle solids under compression, Mech. Mater. 29, 81-92.

Linkov, A. M. and Mogilevskaya, S. G. [1990] Hypersingular integrals in plane problems of the theory of elasticity, Appl. Math. Mech. 54, 93-99.

Linkov, A. M. and Mogilevskaya, S. G. [1994] Complex hypersingular integrals and integral equations in plane elasticity, Acta Mech. 105, 189-205.

Linkov, A. M. [2002] Boundary Integral Equations in Elasticity Theory (Kluwer, Dordrecht).

Martin, P. A. [2000] Perturbed cracks in two dimensions: An integral-equation approach, Int. J. Fract. 104, 317-327.

Mayrhofer, K. and Fischer, F. D. [1992] Derivation of a new analytical solution for a general two-dimensional finite-part integral applicable in fracture mechanics, Int. $J$. Num. Meth. Eng. 33, 1027-1047.

Mogilevskaya, S. G. [2000] Complex hypersingular integral equation for the piece-wise homogeneous half-plane with cracks, Int. J. Fract. 102, 177-204.

Murakami, Y. (ed.) [1987] Stress Intensity Factors Handbook (Pergamon, Oxford).

Muskhelishvili, N. I. [1953] Some Basic Problems of the Mathematical Theory of Elasticity (Noordhoff, Groningen).

Savruk, M. P. [1981] Two-Dimensional Problems of Elasticity for Body with Crack (Nauka Dumka, Kiev) (in Russian).

Woo, C. W., Cheung, Y. K., Chen, Y. Z. and Wang, Y. H. [1988] A simple model for the contact problem of a finite cracked plate in bending, Eng. Fract. Mech. 29, 227-231. 